\title{
Investigation of Methods Based on International Standards to Determine Sound Power of Machinery Noise Sources Under Hemi-anechoic Conditions
}

\author{
Stephen Keith ${ }^{\dagger}$ \\ Radiation Protection Bureau, Health Canada, 775 Brookfield Rd., Ottawa, Ontario, K1A 1C1, Canada \\ G. Krishnappa ${ }^{\dagger}$ \\ Diagnosonics, 4773 Massey Lane, Gloucester, Ontario, K1J 8W8, Canada
}

(Received 11 March 2001; accepted 18 April 2001)

\begin{abstract}
Sound power standards using sound pressure and sound intensity measurements, developed by the International Organisation for Standardisation, were compared on the basis of accuracy and speed. Measurements were carried out under hemi-anechoic conditions on three sources, a reference source, a vacuum cleaner and a circular saw. Both hemispherical and box measurement surfaces were employed in the investigations as prescribed in the standards. The measurement accuracy of the results was examined in two different ways. In the first method the accuracy was assumed to be related to the measurement grade, which was determined by the criteria given in the associated standards. The other method was to find the largest discrepancy between the measured values and the best available estimate. The results for all three sources showed similar trends. The minimum time to obtain a given measurement grade was found for pressure measurements at points distributed over a hemispherical surface, or scanning intensity measurements over a box surface. To obtain the same measurement grade, point intensity measurements over a hemisphere took up to twice the time required by the corresponding pressure measurements. Intensity measurements for points over a box surface took at least twice the time needed for pressure measurements for points on a hemisphere. The investigations also suggested that intensity scanning measurements could be employed to achieve precision grade.
\end{abstract}

${ }^{\dagger}$ Member of the International Institute of Acoustics and Vibration (IIAV)

\section{INTRODUCTION}

The importance of reducing the noise exposure of workers to permissible levels via engineering methods is widely recognised in occupational noise criteria and regulations. To facilitate the use of engineering controls, machinery marketed within European Union (EU) countries must, under the EU Machinery Directive, include noise emission declarations. Two fundamental quantities of sound measurements: sound power levels and emission sound pressure levels are involved in noise emission declarations.

The ISO 11200 series are basic standards that specify methods for measuring emission sound pressure levels at workstations and other specified positions around machinery and equipment. ${ }^{1}$ The ISO $3740^{2}$ series and ISO $9614^{3-5}$ series make up a set of basic standards to determine sound power levels of machinery and equipment. ISO 3741-3747 standards are used to determine sound power levels using sound pressure measurements in specific acoustical environments. The series ISO 9614-1 to ISO 9614-3 describes methods for determining the sound power in almost any environment using sound intensity..$^{3-5}$ Both the series, 3740 and 9614, provide three measurement grades, precision, engineering and survey grades.

The intent of this investigation is to identify sound power measurement standards that are both rapid and accurate. The sound power radiated by a source is determined by integrating the scalar product of the sound intensity vector and the associated elemental area vector over a surface fully enclosing the source. In practice, the shape of the enclosing surface, and the number and distribution of sampling points affects the accuracy and speed of measurements. It can take hours to obtain a large number of samples. An efficient measurement will reduce either the number of samples, or the time per sample. In this study a comparison is made of the speed and accuracy of determining sound power levels of noise sources under hemi-anechoic conditions according to ISO $3745^{6}$ and ISO $3744^{7}$ using sound pressure measurements and ISO 9614-1 to ISO $9614-3^{3-5}$ using sound intensity measurements.

\section{SOUND POWER USING SOUND PRESSURE MEASUREMENTS}

The computation of sound power from sound pressure measurements assumes that the sound power is directly proportional to the mean-square sound pressure when averaged over a surface enclosing the source. Measurement of the spatial average over the surface can be carried out either by using an array of fixed microphone positions or by scanning a single microphone along multiple paths. ISO standards on sound power level determination using sound pressure measurements specify the source characteristics, test environment, qualification procedures, and measurement methods which 